\title{
Study on Target Detection in Mainlobe Jamming with Bistatic MIMO Radar
}

\author{
Qing SUN \\ Air Force Engineering University \\ Xi'an, China \\ sunqing2002@126.com
}

\author{
Junliang JI, Jianfeng TAO \\ Air Force Engineering University \\ Xi’an, China
}

\begin{abstract}
The throwing jamming and ESJ are widely used in ECCM technique domain. The higher power active interference can be received by the main lobe of radar system directly, which would degrade the target detection and tracking performance seriously. This paper proposes the target detection method in the presence mainlobe jamming, which is based on bistatic MIMO radar, through the establishment of the DOD domain filtering; the active jamming in main beam would be suppressed thoroughly. The simulation results show that the algorithm has good interference suppression effect.
\end{abstract}

Keywords-Main lobe jamming; Bistatic MIMO; DOD domain; spatial filter.

\section{INTRODUCTION}

With the use of throwing jamming and the wide use of jamming, high power active jamming directly enters the radar system through the mainlobe of radar antenna, which has a serious impact on the target detection and tracking performance. Especially, the jamming equipment with digital radio frequency storage (DRFM) technology is duplicated, and the radar detection signal is delayed to form false targets, which further increases the signal detection difficulty in the background of radar main lobe interference. Therefore, the performance of the main lobe jamming ability becomes the performance index closely concerned by the radar designer and the user. How to suppress the main lobe interference and realize the target detection and tracking in the main lobe interference background become the urgent problem to be solved in radar design and technical improvement. Key technical issues.

It is found that the difficulty of the main lobe jamming is that the interference is at the same angle as the target, and the angle coordinate is close to or coincident with each other, the main lobe interference is suppressed in the airspace, and even reducing the radar system performance of target detection.

In recent years, the main lobe interference suppression technology has received wide attention in the field of radar. A serial of method are considered in this technique domain, including polarization filtering method[1][2], bistatic radar signal processing technology[3], tracking before detection method of weak target detection technology[4-6], blind source separation technology[7-10] etc. These methods provide a theoretical basis based target detection in the background of lobe interference. However, the array processing technology research mainly consider the problem of interference suppression of single main lobe interference, the polarization domain interference suppression techniques need to rely on the spatial polarization feature database, multistatic radar system need precision time, frequency, space synchronization, tracking before detection performance is susceptible to target maneuver. The blind source separation technology needs sparse receiver distribution, and the frequency of the distributed array can't identify the smart jamming of the main valve.

This paper proposed a target detection method for bistatic MIMO radar with DOD domain anti mainlobe jamming technology. With theoretical analysis and simulation verification, the results indicate that the method proposed in this paper has a strong theoretical significance and application value.

\section{ANALYSIS TO THE SYSTEM MODEL}

MIMO technology has received extensive attention in the field of communication and radar. MIMO array (or subarray) transmits orthogonal waveform, which can improve the transmit beam width, reduce the intercepted Probability of radar, increase the scope of the airspace detection and the scanning speed of radar angular. Meanwhile, through a reasonable array layout, the virtual aperture effect can effectively increase the array angle resolution. All these technical features of MIMO array in radar field have a good prospect of popularization and application.

The bistatic radar transmits the radar detection signal and receives the reflected electromagnetic signal of target by separating the transmitting station and the receiving station in different places (two stations are several tens of kilometers away). The receiving station only receives the electromagnetic signals scattered by target and the bistatic radar system. The geometric relationship between the transmitting station and the receiving station determines that the system is superior to the monostatic radar system in anti-stealth, anti-jamming and antireconnaissance.

Compared with single base equidistant array radar, dualbase MIMO radar possesses innate platform advantages in terms of antenna distribution characteristics, received signal dimension and target dwell time.

- Compared with the equidistant antenna array, the bistatic MIMO radar can use the optimized design of the distributed antenna to obtain better performance to 
the mainlobe airspace filtering so as to achieve the suppression to active/passive interference in mainlobe of the airspace.

- The high-dimensional signals obtained by the bistatic MIMO radar receiver can realize the joint processing of DOA and DOD domain. Compared with the conventional radar, the DOD domain information processing provides a new technical approach for deception jamming detection.

- Bistatic MIMO radar can achieve longer target dwell time by transmitting wide beam, which provides the possibility to improve the target frequency domain resolution for long time processing. Combined with compression sensing technology, sparse detection can be realized in space frequency domain. Target resolution for dense target/passive interference resolution to provide technical support.

In bistatic MIMO radar system, the transmitting array of each element can be independently transmitting orthogonal signals, so the receiver of each array element according to various waveform emitter array were matched filter, can independently obtain the transmit elements to the receiving target scattering signal of the array the output of $\left\{\varepsilon_{R_{i}}, \beta_{R_{i}}, r_{R_{i}}\right\}$ each matched filter containing a transmitting array to the target from the angle of wave, which is called Direction Of Departure(DOD) information. This information can't be changed in the DRFM jammer processing.

We assume that the bistatic MIMO radar system transmitting and receiving arrays are two-dimensional array. The element number of transmitting array is $\mathrm{N}_{\varepsilon_{\mathrm{S}}} \cdot \mathrm{N}_{\beta_{\mathrm{S}}}$, the element number receiving antenna array is $\mathrm{N}_{\varepsilon_{\mathrm{R}}} \cdot \mathrm{N}_{\beta_{\mathrm{R}}}$. As the spacing between the neighboring elements is $\frac{\lambda_{0}}{2}$. The transmitting baseband signal is $s_{m n}(t)$, The number of target and jamming in the far field is $I$, the coordinate of the $i$ th target and interference relative to the transmitting array is $\left\{\varepsilon_{S_{i}}, \beta_{S_{i}}, r_{S_{i}}\right\}$, and the coordinate to the receiving array is relative In the polar coordinates of the receiving array is, the target base band signal output of the receiving array quadrature matched filter output can be expressed as

$\mathbf{r}_{T}(t)=\sum_{i=1}^{I} \rho_{i}(t) \mathbf{a}_{\varepsilon_{S_{i}}}(t) \circ \mathbf{a}_{\beta_{S_{i}}}(t) \circ \mathbf{a}_{\varepsilon_{R_{i}}}(t) \circ \mathbf{a}_{\beta_{R_{i}}}(t)$

where $\odot$ denotes Hadamard product, $\circ$ denotes the outer product of vectors. The directional vector and signal matrix can be expressed as

$$
\begin{aligned}
& \mathbf{a}_{\varepsilon_{R_{i}}}(t)=\left[1, e^{\mathrm{j} \pi \sin \left(\varepsilon_{\mathrm{R}_{\mathrm{i}}}(\mathrm{t})\right)}, \cdots, e^{\mathrm{j}\left(\mathrm{N}_{\varepsilon_{\mathrm{R}}}-1\right) \pi \sin \left(\varepsilon_{\mathrm{R}_{\mathrm{i}}}(\mathrm{t})\right)}\right]^{T} \\
& \mathbf{a}_{\beta_{R_{i}}}(t)=\left[1, e^{\mathrm{j} \pi \sin \left(\beta_{\mathrm{R}_{\mathrm{i}}}(\mathrm{t})\right)}, \cdots, e^{\mathrm{j}\left(\mathrm{N}_{\beta_{\mathrm{R}}}-1\right) \pi \sin \left(\beta_{\mathrm{R}_{\mathrm{i}}}(\mathrm{t})\right)}\right]^{T}
\end{aligned}
$$

$$
\begin{aligned}
& \mathbf{a}_{\varepsilon_{S_{i}}}(t)=\left[1, e^{\mathrm{j} \pi \sin \left(\varepsilon_{\mathrm{S}_{\mathrm{i}}}(\mathrm{t})\right)}, \cdots, e^{\mathrm{j}\left(\mathrm{N}_{\varepsilon_{\mathrm{S}}}-1\right) \pi \sin \left(\varepsilon_{\mathrm{S}_{\mathrm{i}}}(\mathrm{t})\right)}\right]^{T} \\
& \mathbf{a}_{\beta_{S_{i}}}(t)=\left[1, e^{\mathrm{j} \pi \sin \left(\beta_{\mathrm{S}_{\mathrm{i}}}(\mathrm{t})\right)}, \cdots, e^{\mathrm{j}\left(\mathrm{N}_{\beta_{\mathrm{S}}}-1\right) \pi \sin \left(\beta_{\mathrm{S}_{\mathrm{i}}}(\mathrm{t})\right)}\right]^{T}
\end{aligned}
$$

The interference signal and the target signal have the same signal structure, which can be expressed as

$$
\begin{aligned}
\mathbf{r}_{J}(t) & =\sum_{j=1}^{J} \sum_{k=1}^{K_{j}} g_{j k} \mathbf{a}_{\varepsilon_{S_{j k}}}(t) \circ \mathbf{a}_{\beta_{S_{j k}}}(t) \\
& \circ \mathbf{a}_{\varepsilon_{R_{j k}}}(t) \circ \mathbf{a}_{\beta_{R_{j k}}}(t)
\end{aligned}
$$

The output of each receiving array can be expressed as

$$
\mathbf{r}_{R}(t)=\mathbf{r}_{T}(t)+\mathbf{r}_{J}(t)+\mathbf{r}_{N}(t)
$$

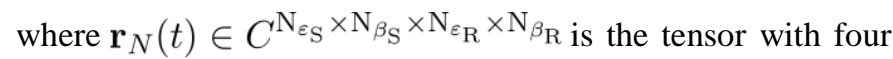
order.

Comparing the bistatic MIMO radar with the monostatic radar, the matched filter output of the receiving would have higher dimension, which would bring convenience to the mainlobe jamming filtering. Therefore, the main lobe antijamming technology, which is based on DOD domain jamming filtering, would have better performance than that of monostatic radar.

\section{THE PROPOSED METHOD}

In bistatic MIMO radar, the interference send from the jammer which is located in the main lobe of the receiving station and the side lobe of the transmitting station can be filtered in the DOD domain to achieve mainlobe jamming suppression. The filtering processing procedure for high order data can be expressed as

$$
\mathbf{y}_{R}(t)=\mathbf{r}_{R}(t) \times_{1} w_{\varepsilon_{S}} \times_{1} w_{\beta_{S}}
$$

where $\times_{1}$ represent product of $n$-mode tensor and matrix, $w_{\varepsilon_{S}}$ and $w_{\beta_{S}}$ represents the beamformer in elevation and azimuth domain, respectively. In order to remove the DOD domain interference, the following optimized mainlobe DOD domain filter is designed as

$$
\begin{aligned}
& \min w^{H} w, \text { s.t. } w^{H} A_{0}=g_{0}^{H} \\
& A_{0}=\left[a_{T}, a_{J_{1}}, a_{J_{2}}, \cdots, a_{J_{N}}\right] \\
& g_{0}=[1,0,0, \cdots, 0]^{H}
\end{aligned}
$$

Among them, $a_{T}$ is for the desired signal steering vector, $a_{J_{i}}$ is for the interference signal oriented vector, the use of Lagrange number of multiplicative solution to this optimization problem 


$$
\begin{aligned}
\xi(w, \lambda) & =w^{H} w+\left(w^{H} A_{0}-g_{0}^{H}\right) \lambda \\
\frac{\partial \xi(w, \lambda)}{\partial w} & =2 w+A_{0} \lambda=0 \\
w^{H} A_{0} & =g_{0}^{H}
\end{aligned}
$$

and the solution is

$$
w=A_{0}\left(A_{0}^{H} A_{0}\right)^{-1} g_{0}
$$

Therefore, the filter in DOD domain at elevation and azimuth directional can be written as:

$$
\begin{aligned}
& w_{\varepsilon_{S}}=A_{\varepsilon_{S_{0}}}\left(A_{\varepsilon_{S_{0}}}^{H} A_{\varepsilon_{S_{0}}}\right)^{-1} g_{\varepsilon_{S_{0}}} \\
& w_{\beta_{S}}=A_{\beta_{S_{0}}}\left(A_{\beta_{S_{0}}}^{H} A_{\beta_{S_{0}}}\right)^{-1} g_{\beta_{S_{0}}}
\end{aligned}
$$

\section{NUMERICAL SIMULATION}

The simulation set a target signal to noise ratio as $20 \mathrm{~dB}$, and the signal to interference ratio is - $3 \mathrm{~dB}$, the interference source is located in the sidelobe in DOD domain, the elevation of the target and jamming angle are 3 and 8 degrees shown in Fig.1, respectively. The azimuth in DOD domain are -28 and 18.5 degrees shown in Fig.2, respectively. The spatial pattern of the optimal DOD domain interference suppression filter as shown in Fig. 3 and Fig. 4.

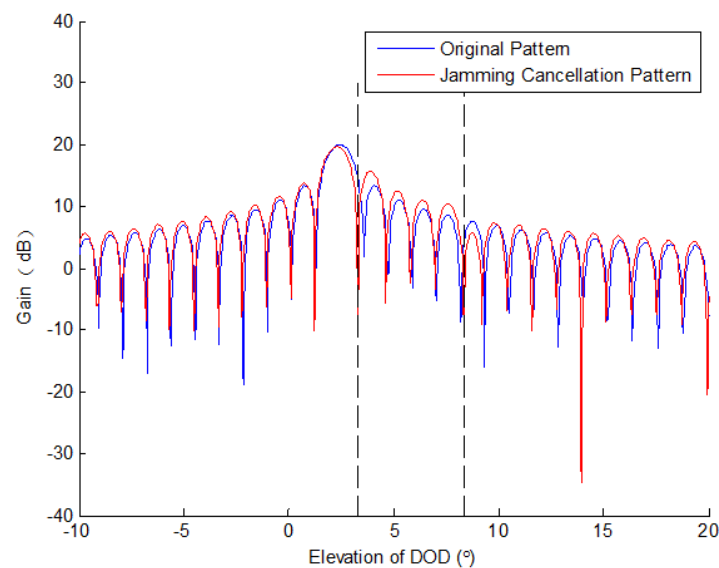

Fig. 1. DOD domain filter on elevation dimension

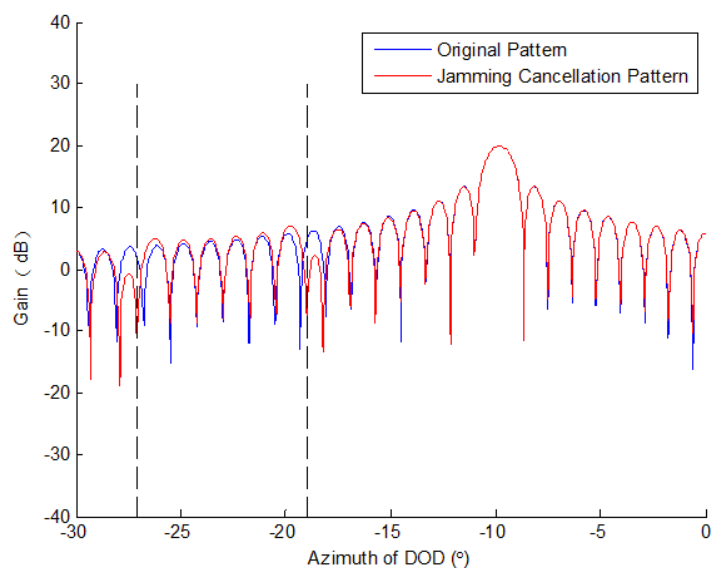

Fig. 2. DOD domain filter on azimuth dimension

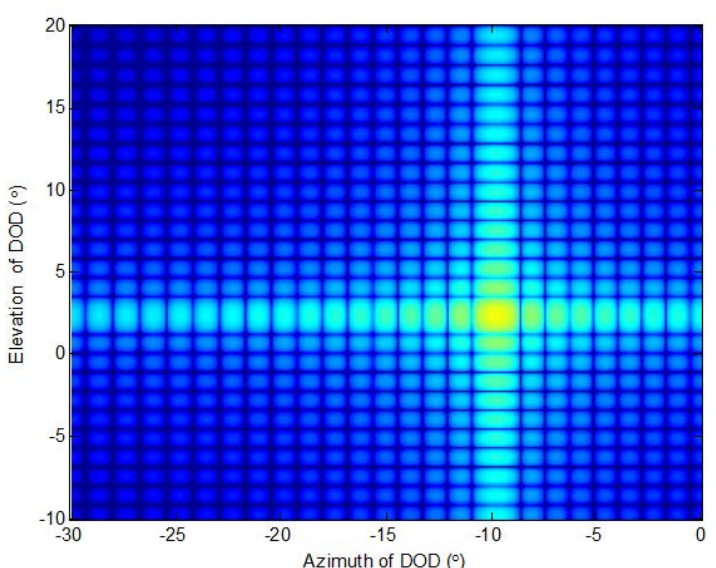

Fig. 3. Original beam pattern

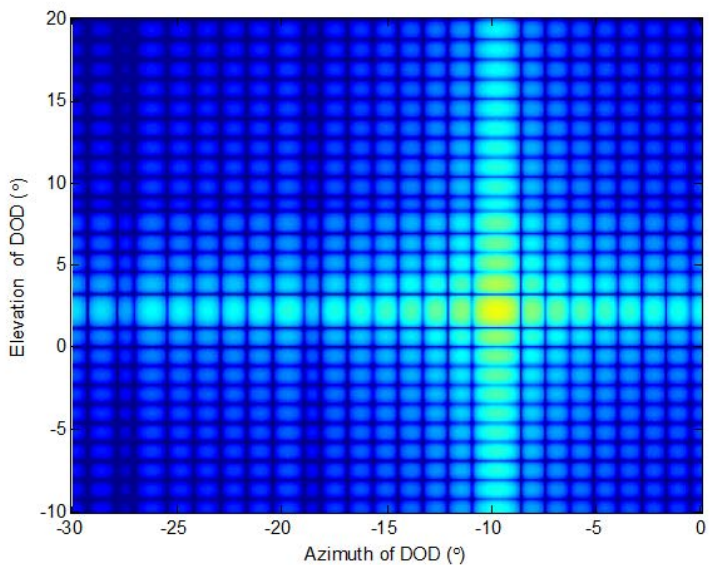

Fig. 4. Proposed beam pattern

The jamming are filtered out in DOD domain, the target signal is reserved by the filter. The range-angular detection result of radar is shown in fig. 5 and fig. 6.

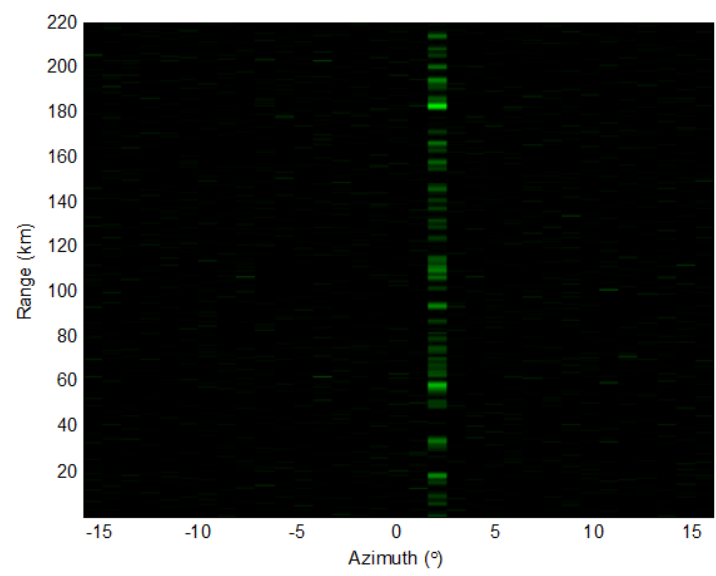

Fig. 5. Original radar screen 


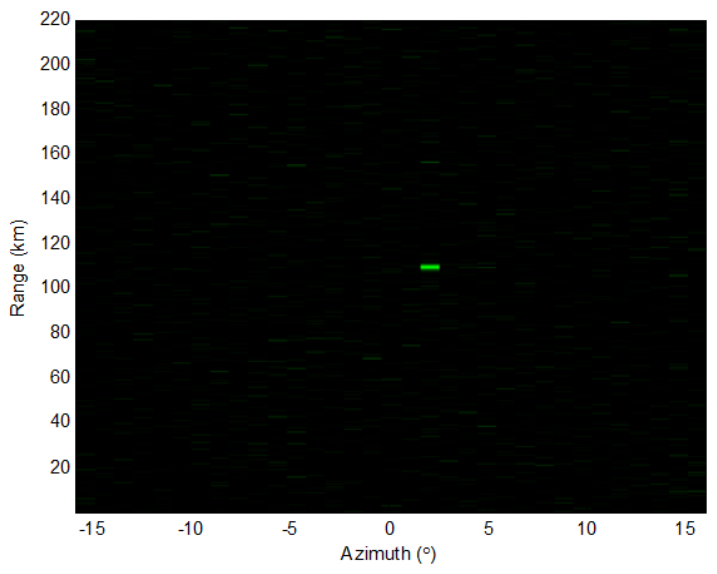

Fig. 6. Radar screen under the proposed method

\section{CONCLUSIONS}

In this paper, a signal detection method based on Bistatic MIMO Radar is considered, and the DOD domain filter is designed. The target signal and jamming signal can be separated by the DOD domain filter. Numerical simulations have verified the effectiveness of this method.

However, when a target is close to the deception jamming and the passive jamming parameters, the bistatic MIMO radar system can't distinguish the dense distribution signal. Therefore, it is necessary to further enhance the resolving ability. The dual-base MIMO radar has wide beam emission characteristics, which provides a platform for long-term detection. Aiming at the long-term target/jamming signal detection, we will do further research to improve the radar target resolution.

\section{REFERENCES}

[1] R.C. DAVIS et al, “Angle Estimation With Adaptive Arrays in External Noise Fields," IEEE Transactions on Aerospace and Electronic Systems.pp.179-186,Mar.1976.

[2] Michael D. Zoltowski, Martin Haardt and Cherian P. Mathews, "Closed form 2-D angle estimation with rectangular arrays in element space of beamspace via unitary ESPRIT”IEEE Transaction on Signal Processing, vol. 44,pp. 316-328, 1996.

[3] Victor S Chernyak, “Adaptive mainlobe jamming cancellation and target detection in multistatic radar systems,"CIE International Conference of Radar Proceedings pp.297-300,Oct.1996.

[4] R.P. Perry, et al, "Coherent Integration With Range Migration Using Keystone Formatting,”IEEE Radar Conference,2007,pp.683-868.

[5] Pang Cunsuo and Ran Tao,“A High Speed Target Detection Approach Based on STFrFT,"2011 International Conference on Instrumentation,Measurement,Computer,Communication and Control, 2011, pp. 744-47.

[6] Xiaolong Chen,Jian Guan,Ningbo Liu and You He, "Maneuvering Target Detection via Radon-Fractional Fourier Transform-Based LongTime Coherent Integration,”IEEE Transactions on signal processing, vol.62, pp. 939-953, 2014.

[7] C.Simon ,Ph.Loubaton and C. Jutten, "Separation of a class of convolutive mixtures: a contrast function approach Signal Processing”, vol.81, pp. 883-887, 2001.

[8] Marc Castella and Eric Moreau "New kurtosis optimization schemes for MISO equalization” IEEE Transactions on signal processing”, vol.60, pp. 1319-1330, 2012.

[9] Marc Castella, Saloua Rhioui,Eric Moreau and Jean-Christophe Pesquet "Quadratic higher-order criteria for iterative blind separation of a MIMO convolutive mixture of sources" IEEE Transactions on signal processing”, vol.55, pp. 1-15, 2007.

[10] Marc Castella and Eric Moreau“Generalized identifiability conditions for blind convolutive MIMO separation” IEEE Transactions on signal processing”, vol.57, pp. 2846-2852, 2009. 